Bundesgesundheitsbl 2015 · 58:769-773

DOI 10.1007/s00103-015-2175-9

Online publiziert: 28. Mai 2015

c) Springer-Verlag Berlin Heidelberg 2015

Bekanntmachung des Umweltbundesamtes

\title{
Gesundheitliche Bewertung krebserzeugender Verunreinigungen der Innenraumluft - erste Ergänzung zum Basisschema
}

\author{
Mitteilung des Ausschusses für \\ Innenraumrichtwerte
}

\section{Einleitung}

Zur gesundheitlichen Bewertung von Verunreinigungen der Innenraumluft ist nach dem Basisschema [1] zunächst der empfindlichste toxische Endpunkt (kritische Effekt) zu identifizieren. Falls sich dabei eine krebserzeugende Wirkung als empfindlichster Endpunkt erweist oder bei einem anderen empfindlichsten Endpunkt eine kanzerogene oder gentoxische Wirkung beteiligt ist, ist die toxikologische Bewertung dieses Stoffes auf der Grundlage der krebserzeugenden Wirkung vorzunehmen. Das dabei anzuwendende Verfahren wird in dieser Mitteilung dargestellt.

\section{Stoffauswahl}

Der Ausschuss für Innenraumrichtwerte wählt die nach diesem Verfahren zu berücksichtigenden Stoffe anhand von zwei Kriterien aus: anhand einer Einstufung eines Stoffes als krebserzeugend und im Hinblick auf ein Vorkommen dieses krebserzeugenden Stoffes in der Innenraumluft.

Hinsichtlich der Einstufung eines Stoffes als krebserzeugend greift der Ausschuss auf die von der Deutschen Gesetzlichen Unfallversicherung aus dem europäischen und deutschen Gefahrstoffrecht zusammengestellte und regelmäßig aktualisierte sogenannte KMR-Liste [2] zu- rück. Diese Liste enthält alle Stoffe, die in eine der drei Kanzerogenitätskategorien 1A, $1 \mathrm{~B}$ oder 2 eingestuft sind ( $\bullet$ Tab. 1). Um möglichen Missverständnissen vorzubeugen, sei darauf hingewiesen, dass die Einstufung in die jeweilige Kategorie auf Grund der Datenlage erfolgt und weder die Wirkstärke noch die Höhe eines Krebsrisikos abbildet.

Der Ausschuss berücksichtigt gegebenenfalls auch neue, noch nicht rechtlich verbindliche Einstufungsvorschläge des Risikobewertungsausschusses der Europäischen Chemikalienbehörde (ECHARAC) oder der Arbeitsstoff-Kommission der Deutschen Forschungsgemeinschaft. Einbezogen in diese Stoffauswahl wird auch das Radon, das in den Leitlinien der Weltgesundheitsorganisation zur Innenraumluftqualität aufgeführt ist und in vielen Gebäuden in Deutschland für nichtrauchende Personen die gesundheitlich bedeutendste krebserzeugende Verunreinigung der Innenraumluft darstellt [4].

Als weiteren Schritt prüft der Ausschuss, ob einer dieser als krebserzeugend eingestuften Stoffe bereits in der Luft von Innenräumen einschließlich Innenraumarbeitsplätzen (ohne Tätigkeiten mit Gefahrstoffen) nachgewiesen wurde.

\section{Anforderungen an Daten zur Risikobewertung}

\subsection{Daten zum Vorkommen krebserzeugender Stoffe in der Innenraumluft}

Umfangreiche oder sogar repräsentative Messergebnisse liegen in Deutschland nur für eine überschaubare Anzahl krebserzeugender Stoffe in der Innenraumluft vor, zusätzlich sind für einige weitere Stoffe Einzeldaten oder Angaben aus anderen Ländern in der Literatur verfügbar. Als Mindestangaben zum Vorkommen krebserzeugender Stoffe in der Innenraumluft sollten das 50. und das 95. Perzentil (üblicherweise als Referenzwert bezeichnet [5]) sowie der Maximalwert verfügbar sein. Bevorzugt werden Angaben, die repräsentativ für Deutschland sind.

Beispielsweise wurde das Vorkommen einiger flüchtiger und sehr flüchtiger organischer krebserzeugender Verbindungen in der Innenraumluft im Kinder-Umwelt-Survey von 2003 bis 2006 deutschlandweit repräsentativ erhoben [6]. Dabei handelt es sich um Ergebnisse von einwöchigen Messungen unter Nutzungsbedingungen mithilfe von Passivsammlern. Umfangreiche Ergebnisse mit Angaben zum 50. und zum 95. Perzentil finden sich auch in den Auswertungen von Kurzzeitmessungen der Arbeitsgemeinschaft der Ökologischen Forschungsinstitute (AG- 
Tab. 1 Kategorien der krebserzeugenden Wirkung laut Verordnung (EG) Nr. 1272/2008 [3]

\begin{tabular}{|c|c|}
\hline Kategorie & Beschreibung \\
\hline Karz. 1 & $\begin{array}{l}\text { Bekanntermaßen oder wahrscheinlich beim Menschen karzinogen. Ein Stoff wird } \\
\text { anhand epidemiologischer und/oder Tierversuchsdaten als karzinogen der Kate- } \\
\text { gorie } 1 \text { eingestuft. Die Einstufung eines Stoffes kann weiter wie folgt differenziert } \\
\text { werden: }\end{array}$ \\
\hline Karz. 1A & $\begin{array}{l}\text { Stoffe, die bekanntermaßen beim Menschen karzinogen sind; die Einstufung er- } \\
\text { folgt überwiegend aufgrund von Nachweisen beim Menschen }\end{array}$ \\
\hline Karz. 1B & $\begin{array}{l}\text { Stoffe, die wahrscheinlich beim Menschen karzinogen sind; die Einstufung erfolgt } \\
\text { überwiegend aufgrund von Nachweisen bei Tieren. Die Einstufung in Kategorie } \\
\text { 1A und 1B beruht auf der Aussagekraft der Nachweise in Verbindung mit zusätz- } \\
\text { lichen Hinweisen. Diese Nachweise können entweder aus epidemiologischen } \\
\text { Studien, die einen ursächlichen Zusammenhang zwischen der Exposition von } \\
\text { Menschen gegenüber einem Stoff und der Entwicklung von Krebs nahe legen } \\
\text { (bekanntes Humankarzinogen), oder aus Tierversuchen stammen, deren Beweis- } \\
\text { kraft ausreicht, eine karzinogene Wirkung beim Tier (wahrscheinliches Human- } \\
\text { karzinogen) nachzuweisen. Darüber hinaus kann es im Einzelfall aufgrund einer } \\
\text { wissenschaftlichen Beurteilung gerechtfertigt sein, eine Entscheidung über die } \\
\text { wahrscheinliche karzinogene Wirkung beim Menschen auf Untersuchungen zu } \\
\text { stützen, die nur begrenzte Nachweise auf eine karzinogene Wirkung beim Men- } \\
\text { schen in Verbindung mit begrenzten Nachweisen bei Versuchstieren ergaben }\end{array}$ \\
\hline Karz. 2 & $\begin{array}{l}\text { Verdacht auf karzinogene Wirkung beim Menschen. Die Einstufung eines Stoffes } \\
\text { in Kategorie } 2 \text { erfolgt aufgrund von Nachweisen aus Studien an Mensch und/oder } \\
\text { Tier, die jedoch nicht hinreichend genug für eine Einstufung des Stoffes in Kate- } \\
\text { gorie } 1 \text { A oder } 1 B \text { sind, anhand der Aussagekraft der Nachweise und zusätzlicher } \\
\text { Hinweise. Solche Nachweise können entweder aus Studien beim Menschen, die } \\
\text { einen Verdacht auf karzinogene Wirkung begründen, oder aus Tierstudien, die } \\
\text { einen Verdacht auf karzinogene Wirkungen ergeben, stammen }\end{array}$ \\
\hline
\end{tabular}

ÖF) aus 2002 bis 2006 [7]. Allerdings ist unklar, ob diese Daten für Deutschland als repräsentativ angesehen werden können, da die Proben in der Regel anlassbezogen genommen worden waren und der AGÖF-Bericht keine Angaben zur regionalen Herkunft der Proben enthält.

Ergebnisse von Messungen über einen längeren Zeitraum (mindestens drei Monate bis zu einem Jahr) liegen nur für Radon in der Innenraumluft gemäß DIN ISO 11665 Blatt 8 [8] vor.

Aus Sicht des Ausschusses ist es unverzichtbar, dass auch weiterhin repräsentative Untersuchungen zum Vorkommen krebserzeugender Stoffe in der Innenraumluft durchgeführt werden, aus denen aktuelle Referenzwerte abgeleitet werden können.

\subsection{Angaben zum Risiko durch krebserzeugende Stoffe in der Innenraumluft}

Mögliche gesundheitliche Risiken durch eingeatmete krebserzeugende Stoffe lassen sich mit Hilfe mathematischer Modelle abschätzen. Auch wenn derartige Risikoabschätzungen wissenschaftlich nach wie vor nicht unumstritten sind, werden sie inzwischen von regulatorischen Gremien auf internationaler Ebene (z. B. von Expertengremien der Weltgesundheitsorganisation), auf europäischer Ebene (z. B. von der Europäischen Chemikalienbehörde - ECHA -) und auf nationaler Ebene (z. B. vom Ausschuss für Gefahrstoffe) zunehmend vorgenommen.

Als Datengrundlage dienen im Idealfall bevölkerungsbezogene Studien in dem interessierenden Konzentrationsbereich. Solche Studien sind aber nur für sehr wenige Stoffe verfügbar. Weitaus häufiger liegen lediglich Beobachtungen an Arbeitsplätzen oder tierexperimentelle Untersuchungen bei sehr hohen Expositionskonzentrationen vor, aus denen auf eine Expositionssituation mit vergleichsweise niedrigen Stoffkonzentrationen in der Innenraumluft extrapoliert werden muss. Risikoabschätzungen aus Humanstudien sind tierexperimentellen Studien vorzuziehen, da bei letzteren nicht sicher beurteilt werden kann, ob z. B. ein beim Tier gefundener Tumor angesichts bestimmter Unterschiede (z. B. hinsichtlich des Verabreichungsweges, der Enzymausstattung, des Metabolismus oder der To- xikokinetik) möglicherweise einen speziesspezifischen und damit für den Menschen nicht relevanten Befund darstellt.

Die aus Hochdosisstudien auf eine sogenannte lebenslange Exposition (übliche Annahme: 70 Jahre) abgeschätzten theoretischen Krebsrisiken sind nicht als die Anzahl von Krebsfällen zu verstehen, die eine Substanz bei einer bestimmten Konzentration tatsächlich verursachen wird. Nach Ansicht der Weltgesundheitsorganisation handelt es sich vielmehr um das maximal mögliche Risiko, dass bei der bestimmten Konzentration eine Krebserkrankung auftritt. Es ist sehr wahrscheinlich, dass das tatsächliche Risiko niedriger ist, möglicherweise sogar gegen null tendiert [9].

Die Annahme, dass das Krebsrisiko sich null nähern oder nicht von null unterscheidbar sein könnte, gründet sich unter anderem auf die Unsicherheiten, die mit dem Mechanismus der Krebsentstehung und mit möglichen Entgiftungsund Reparaturmechanismen verknüpft sein können. Für die meisten in der Innenraumluft gefundenen krebserzeugenden Stoffe kann deshalb die Frage, ob mit einer bestimmten Konzentration ein nennenswertes Krebsrisiko verknüpft ist oder ob das theoretische Krebsrisiko bei null liegt, nicht beantwortet werden, da die abgeschätzten theoretischen Risiken unterhalb üblicher epidemiologischer Nachweisgrenzen ${ }^{1}$ liegen und deshalb in bevölkerungsbezogenen Studien nicht verifiziert werden können.

Für die Extrapolation von sehr hohen tierexperimentell verwendeten Dosen oder von Arbeitsplatzexpositionen zu sehr niedrigen Expositionen in Innenräumen stehen mehrere Modelloptionen zur Verfügung, z. B. ein linearisiertes oder ein sublineares Extrapolationsmodell.

\footnotetext{
1 In bevölkerungsbezogenen Studien lassen sich Krebsrisiken unter 1:1000 nur mit einem erheblichen (messtechnischen, logistischen und finanziellen) Aufwand entdecken. Beispielsweise konnte in der größten deutschen Studie zum Krebsrisiko durch Radon in der Innenraumluft ein signifikant erhöhtes Risiko für Lungenkrebs nach lebenslanger Exposition ab $100 \mathrm{~Bq}$ Radon $/ \mathrm{m}^{3}$ nachgewiesen werden; diese Konzentration entspricht einer epidemiologischen Nachweisgrenze des Krebsrisikos von Nichtrauchern von etwa 1: 1700 $\left(6 \times 10^{-4}\right)[4,10]$.
} 
Ein linearisiertes Modell wird vor allem beim Fehlen von hinreichenden Kenntnissen zum Wirkungsmechanismus der Krebsentstehung angenommen. Angesichts der oben dargestellten Gründe kann die Verwendung eines linearisierten Modells das theoretische Krebsrisiko überschätzen. ${ }^{2}$ Aus Gründen der Vorsorge wird eine Überschätzung des Risikos aber hingenommen.

Ein sublinearer Verlauf eines theoretischen Krebsrisikos kann bei solchen gentoxischen krebserzeugenden Stoffe zugrunde gelegt werden, bei denen oberhalb einer bestimmten Konzentration eine (zyto)toxische Wirkung die krebserzeugende Wirkung verstärkt, während bei Konzentrationen unterhalb dieser (zyto)toxischen Wirkung ein deutlich geringeres Krebsrisiko angenommen wird [11, 12]. Nach einem Vorschlag des Ausschusses für Gefahrstoffe [11], der auch von der ECHA [12] verwendet wird, wird ein sublinearer Verlauf der Expositions-Risikokurve dadurch erzeugt, dass die am NOAEC der (zyto)toxischen Wirkung abgeschätzte Risikohöhe um eine Größenordnung abgesenkt wird. Da sich damit ein abgeknickter Verlauf der Risikofunktion ergibt, wird diese NOAEC auch als Knickpunkt bezeichnet. Im Vergleich zum linearisierten Modell steigt das Risiko oberhalb des Knickpunktes stärker an, während es unterhalb des Knickpunktes etwas abnimmt. Das daraus abgeschätzte theoretische Risiko ist somit im Vergleich zum linearisierten Modell im unteren Dosisbereich weniger konservativ, dürfte aber als Ergebnis einer realitätsnäheren Anpassung des Kurvenverlaufs betrachtet werden. Vor diesem Hintergrund prüft der Ausschuss für Innenraumrichtwerte, welcher Wirkungsmechanismus für eine krebserzeugende Substanz vorliegt und ob ein linearisiertes oder ein sublineares Extrapolationsmodell bevorzugt herangezogen werden sollte.

Bei einigen an Mensch und Tier gut untersuchten krebserzeugenden Stoffen liegen mehrere Angaben zum theoretischen Risiko vor. In diesem Fall prüft der

2 Für einzelne Stoffe wie z. B. Naphthalin konnte diese vermutete Überschätzung anhand der tatsächlich aufgetretenen Krebsfälle aufgezeigt werden [14].

Bundesgesundheitsbl 2015 · 58:769-773 DOI 10.1007/s00103-015-2175-9

(c) Springer-Verlag Berlin Heidelberg 2015

Bekanntmachung des Umweltbundesamtes

\section{Gesundheitliche Bewertung krebserzeugender Verunreinigungen der Innenraumluft: erste Ergänzung zum Basisschema. Mitteilung des Ausschusses für Innenraumrichtwerte}

\section{Zusammenfassung}

Nach Auffassung des Ausschusses für Innenraumrichtwerte müssen zur gesundheitlichen Bewertung eines krebserzeugenden Stoffes in der Innenraumluft belastbare Angaben zu seinem üblichen Vorkommen in der Innenraumluft in Form des 95. Perzentils (Referenzwert) sowie zur Expositions-Risikobeziehung vorliegen. In Abhängigkeit vom Wirkungsmechanismus des krebserzeugenden Stoffes bewertet der Ausschuss, welche Risikofunktion angemessen ist.

Wenn eine belastbare Expositions-Risikobeziehung verfügbar ist, soll diejenige Konzentration des krebserzeugenden Stoffes in der Innenraumluft ermittelt werden, die nach lebenslanger Exposition mit einem theoretischen Krebsrisiko von $10^{-6}$ verbunden ist.
Diese Konzentration soll mit seinem Referenzwert verglichen werden.

Liegt die aus der Expositions-Risikobeziehung abgeleitete Konzentration oberhalb des möglichst aktuellen Referenzwertes, wird sie als risikobezogener Leitwert für die Bewertung verwendet. Ist die Konzentration des Referenzwertes mit einem höheren theoretischen Risiko als $10^{-6}$ verknüpft, wird ein vorläufiger Leitwert anhand des Referenzwertes in der Innenraumluft festgelegt.

\section{Schlüsselwörter}

Kanzerogen · Innenraumluft ·

Expositions-Risikobeziehung · Referenzwert . Risikobezogener Leitwert

\section{Health evaluation of indoor air carcinogens: first addendum to the basic scheme. Communication from the German Committee on Indoor Guidelines}

If the concentration obtained from the exposure-risk relationship exceeds the reference value, it will be used as a risk-related guideline. If the concentration of the reference value is associated with a cancer risk above $10^{-6}$, the reference value will be used as preliminary guideline.

\section{Keywords}

Carcinogen · Indoor air .

Exposure-risk relationship - Reference value . Risk-related guideline
Ausschuss, ob die zugrundeliegenden Studien belastbar sind und die Auswahl der verwendeten Extrapolationsfaktoren und Modellannahmen nachvollziehbar begründet ist. Der Ausschuss behält sich vor, auch eigene Risikoabschätzungen durchzuführen.

Falls sich herausstellen sollte, dass der Kenntnisstand nicht ausreicht, eine Abschätzung des Risikos mithilfe der vorstehend genannten Modelle vorzunehmen, prüft der Ausschuss weitere Verfahren.
Zur groben Abschätzung eines theoretischen Krebsrisikos hat die ECHA vereinfachte Verfahren wie z. B. das sogenannte large assessment factor-Verfahren entwickelt [13]. Derartige Verfahren gehen von einer bestimmten experimentellen oder modellierten Effektdosis aus und verwenden einen festen Extrapolationsfaktor. Wegen der sehr schematischen Herangehensweise sind diese Verfahren mit noch größeren Unsicherheiten als die davor genannten Verfahren behaftet. Nach 
Auffassung des Ausschusses ermöglichen sie lediglich einen ersten Hinweis auf eine theoretische Risikohöhe.

\section{Gesundheitliche Bewertung des Risikos durch krebserzeugende Stoffe der Innenraumluft}

Nach Auffassung der Gesundheitsministerkonferenz [15] kann die Frage, welches Risiko für die Bevölkerung als hinnehmbar („tolerabel“) oder zulässig (,akzeptabel") anzusehen ist, nicht allein auf der Ebene der Verwaltung beantwortet werden. Solche Risikofestsetzungen setzen vielmehr einen gesellschaftlichen Meinungsbildungsprozess voraus und obliegen damit letztlich den zuständigen europäischen oder deutschen parlamentarischen Organen und deren beratenden wissenschaftlichen Gremien.

Vor diesem Hintergrund sieht es der Ausschuss für Innenraumrichtwerte als seine Aufgabe an, den Kenntnisstand zur gesundheitlichen Wirkung krebserzeugender Stoffe in der Innenraumluft darzustellen und auf die mit den vorstehend genannten Risikomodellen abgeschätzten theoretischen Krebsrisiken dieser Stoffe bei bestimmten Konzentrationen hinzuweisen. Die Krebsrisiken der zurzeit in der Innenraumluft von Gebäuden in Deutschland anzutreffenden krebserzeugenden Verunreinigungen weisen unter der Annahme einer lebenslangen Exposition eine außerordentliche Breite auf. Das theoretische Krebsrisiko einzelner Noxen reicht dabei von unter 1:100.000.000 (in Potenzform angegeben: $10^{-8}$ ) bis über 1:100 $\left(10^{-2}\right)$ (Radon) [4]. Angesichts dieser Risikobreite erscheint es dem Ausschuss sowohl im Hinblick auf die Dringlichkeit von Maßnahmen (Prioritätensetzung) als auch auf eine Risikokommunikation angemessen, ein gestuftes Verfahren zur Verringerung der Exposition gegenüber krebserzeugenden Stoffen in der Innenraumluft zu entwickeln.

In ihrem Leitfaden zur Risikoabschätzung empfiehlt die ECHA, bei Abschätzungen der Exposition der Allgemeinbevölkerung angesichts des Fehlens gesetzlicher Regelungen ein sogenanntes indikatives Risiko von $10^{-6}$ anzusetzen [13]. Auch die Weltgesundheitsorganisation nennt in ihren Risikoabschätzungen $10^{-6}$ als unteren Wert eines möglichen Risikobereiches $\left(10^{-4}\right.$ bis $\left.10^{-6}\right)$ [4].

Unter der Voraussetzung, dass eine Abschätzung des theoretischen Krebsrisikos einer Verunreinigung der Innenraumluft als belastbar angesehen werden kann und der Referenzwert dieser Verunreinigung in der Innenraumluft bekannt ist, empfiehlt der Ausschuss folgendes Vorgehen:

Wenn die üblicherweise vorkommende Konzentration einer krebserzeugenden Verunreinigung in der Innenraumluft, dargestellt durch ihren Referenzwert, bei Annahme einer lebenslangen Exposition ein theoretisches Krebsrisiko von 1:1.000.000 $\left(10^{-6}\right)$ unterschreitet, erscheint es dem Ausschuss beim derzeitigen Kenntnisstand unverhältnismäßig, bei einer solchen Konzentration expositionsmindernde Maßnahmen zu ergreifen. In diesem Fall bezeichnet der Ausschuss die mit einem theoretischen Krebsrisiko von $10^{-6}$ verbundene Konzentration als risikobezogenen Leitwert.

Wenn die üblicherweise vorkommende Konzentration einer krebserzeugenden Verunreinigung in der Innenraumluft, dargestellt durch ihren Referenzwert, bei Annahme einer lebenslangen Exposition ein theoretisches Krebsrisiko von 1:1.000.000 $\left(10^{-6}\right)$ übersteigt, benennt der Ausschuss in einem solchen Fall keinen risikobezogenen Leitwert, sondern verwendet den Referenzwert (das 95. Perzentil einer für Deutschland repräsentativen Stichprobe) als vorläufigen Leitwert. Dieser ist als ein rein technischer Wert anzusehen. Bei Konzentrationen oberhalb dieses vorläufigen Leitwertes eines krebserzeugenden Stoffes sollten grundsätzlich expositionsmindernde Maßnahmen ergriffen werden. Im Hinblick auf eine Prioritätensetzung informiert der Ausschuss über das mit dem vorläufigen Leitwert einer krebserzeugenden Verunreinigung in der Innenraumluft nach lebenslanger Exposition verbundene theoretische Krebsrisiko.

Der verwendete Referenzwert soll regelmäßig (etwa alle 5 Jahre) überprüft und der vorläufige Leitwert gegebenenfalls angepasst, d. h. wenn möglich, abgesenkt werden. Falls der Referenzwert eines Stoffes in der Innenraumluft bei einer
Überprüfung angestiegen sein sollte, soll der vorläufige Leitwert im Sinne einer Minimierungsstrategie unverändert bleiben.

Der Ausschuss wird in seinen Mitteilungen zu den einzelnen krebserzeugenden Stoffen in der Innenraumluft die jeweiligen Abschätzungen zum risikobezogenen Leitwert und den aktuellen Referenzwert darstellen. Darüber hinaus sollen in den Stoffberichten Hinweise zu Quellen möglicher Belastungen enthalten sein. Im Falle einer Überschreitung des risikobezogenen beziehungsweise vorläufigen Leitwertes können diese als Ansatzpunkt für Minimierungsstrategien dienen. Im Falle einer Erhöhung des 95. Perzentils bei einer repräsentativen Datenerhebung sollten die Quellenhinweise aktualisiert werden.

Beim derzeitigen Kenntnisstand [4] ist darauf hinzuweisen, dass bei einzelnen krebserzeugenden Stoffen in der Innenraumluft expositionsmindernde Maßnahmen aus verschiedenen Gründen nur in einem begrenzten Umfang möglich oder sinnvoll sind. In Einzelfällen werden wir in Deutschland gegebenenfalls auf längere Zeit mit einem höheren Krebsrisiko leben müssen als durch einen risikobezogenen Leitwert in der Innenraumluft vorgegeben wäre. Beispielsweise beträgt für lebenslang in Deutschland lebende Nichtraucher das vor allem durch Radon in der Innenraumluft bedingte, unvermeidbare Lungenkrebsrisiko über $10^{-4}$ [4].

Für einige krebserzeugende Verunreinigungen in der Innenraumluft erfolgt ein Eintrag über die Außenluft. Daher sollte der Eintrag aus der Außenluft mit dargestellt werden. Im Einzelfall kann es vorkommen, dass keine weitere Minimierung der krebserzeugenden Verunreinigung in der Innenraumluft möglich ist, da sich die Konzentration schon auf dem Niveau des Außenlufteintrages befindet. Generell gilt aber die maximal zulässige Außenluftkonzentration nicht als Beurteilungsgrundlage für die Innenraumluftkonzentration eines kanzerogenen Stoffes.

Schließlich ist auch grundsätzlich möglich, dass eine vorliegende endogene Konzentration einer krebserzeugenden Substanz im Körper des Menschen primär expositionsmindernde Maßnahmen nicht sinnvoll erscheinen lässt [16]. 
Da es sich beim Krebsrisiko durch Verunreinigungen der Innenraumluft in der Regel um ein Risiko nach Langzeitexposition handelt, stellt sich die Frage der Messdauer bei krebserzeugenden Stoffen in der Innenraumluft. Hinweise zur Messstrategie und gegebenenfalls zu Anforderungen an die Bestimmungsgrenze eines krebserzeugenden Stoffes in der Innenraumluft sollen in den Mitteilungen zu den einzelnen krebserzeugenden Stoffen in der Innenraumluft gegeben werden.

\section{Anmerkungen}

Der Entwurf dieser Mitteilung wurde von Dr. Helmut Sagunski, Dr. Martin Kraft und Dr. Hermann Fromme mit Beiträgen von Herrn Herbert Grams, Dr. Birger Heinzow, Dr. Bernhard Link, Herrn Thomas Lahrz, Dr. Susanne Rudzok und Dr. Jutta Witten erstellt und von der Adhoc-Arbeitsgruppe im April 2015 verabschiedet.

\section{Literatur}

1. Ad-hoc-Arbeitsgruppe Innenraumrichtwerte (2012) Richtwerte für die Innenraumluft: erste Fortschreibung des Basisschemas. Bundesgesundheitsblatt 55:279-290

2. DGUV (2014) KMR-Liste. http://publikationen. dguv.de/dguv/pdf/10002/kmr_neue_bezeichnung_2014.pdf Zugegriffen: 30. Jan. 2015

3. Verordnung (EG) Nr. 1272/2008 des Europäischen Parlaments und des Rates vom 16. Dezember 2008 über die Einstufung, Kennzeichnung und Verpackung von Stoffen und Gemischen, zur Änderung und Aufhebung der Richtlinien 67/548/EWG und 1999/45/EG und zur Änderung der Verordnung (EG) Nr. 1907/2006. ABI. L 353 vom 31.12.2008

4. WHO (2010) WHO indoor air quality guidelines selected pollutants. World Health Organization, Geneve

5. Ad-hoc-Arbeitsgruppe der IRK/AOLG (2007) Beurteilung von Innenraumluftkontaminationen mittels Referenz- und Richtwerten. Bundesgesundheitsblatt 50:990-1005

6. UBA (2008) Vergleichswerte für flüchtige organische Verbindungen (VOC und Aldehyde) in Innenraumluft von Haushalten in Deutschland. Ergebnisse des repräsentativen Kinder-Umwelt-Surveys (KUS) des Umweltbundesamtes. Bundesgesundheitsblatt 51:109-112

7. Hofmann H, Plieninger P (2008) Bereitstellung einer Datenbank zum Vorkommen von flüchtigen organischen Verbindungen in der Innenraumluft. Umweltbundesamt. FB 20561234 http://www. umweltbundesamt.de/sites/default/files/medien/ publikation/long/3637.pdf

8. DIN ISO 11665-8 (2013) Ermittlung der Radioaktivität in der Umwelt - Luft. Teil 8: Radon-222 in Gebäuden. Beuth-Verlag, Berlin

9. WHO (2011) Drinking water quality guidelines. World Health Organization, Geneve
10. BfS (2014) Radon. Bundesamt für Strahlenschutz. http://www.bfs.de/de/ion/anthropg/radon/radon_wirkungen.html Zugegriffen: 30. Jan. 2015

11. AGS (2008) Begründung zu Expositions-Risiko-Beziehung für Trichlorethen in BekGS910. Ausschuss für Gefahrstoffe. http://www.baua.de/de/Themenvon-A-Z/Gefahrstoffe/TRGS/Begruendungen-910. html;jsessionid =21B49E5B1BC6BC83E21E4577845 16DEF.1_cid343. Zugegriffen: 21. Jan. 2015

12. ECHA (2014) Application for authorisation: establishing a reference dose response relationship for carcinogenicity of trichlorethylene. RAC/28/2014/07 rev 2 final. European Chemicals Agency. Committee for Risk Assessment. http://echa.europa.eu/documents/10162/13641/carcinogenicity_dose_response_tce_en.pdf. Zugegriffen: 30.Jan. 2015

13. ECHA (2010) Guidance on information requirements and chemical safety assessment. Chapter R.8: Characterisation of dose [concentration]-response for human health. Version: 2. December 2010. Europäische Chemikalienagentur. http://guidance.echa.europa.eu/docs/guidance_document/information_requirements_r8_ en.pdf?vers=16_12_10.Zugegriffen: 30. Jan. 2015

14. Magee B, Samuelian J, Haines K et al (2010) Screening-level population risk assessment of nasal tumours in the US due to naphthalene exposure. Regul Toxicol Pharmacol 51:168-180

15. GMK (1994) Entschließung der Gesundheitsministerkonferenz vom 17./18. November 1994 zum Stellenwert quantitativer Risikoabschätzungen im umweltbezogenen Gesundheitsschutz. In: Ausschuss für Umwelthygiene (1994): Standards zur Expositionsabschätzung. Anhang 2. Behörde für Arbeit, Gesundheit und Soziales, Hamburg (Hrsg.). http://www.apug.de/archiv/pdf/Expositionsabschaetzung_Laender_1995.pdf. Zugegriffen: 30. Jan. 2015

16. AGS (2014) Technische Regel für Gefahrstoffe 910 Risikobezogenes Maßnahmenkonzept für Tätigkeiten mit krebserzeugenden Gefahrstoffen (TRGS910). http://www.baua.de/de/Themen-vonA-Z/Gefahrstoffe/TRGS/TRGS-910.html. Zugegriffen: 30. Jan. 2015 